\title{
LA ÉTICA DEL CUIDADO: CONTRIBUCIONES A UNA TRANSFORMACIÓN PACIFICA DE LOS CONFLICTOS
}

\author{
IRENE COMINS MINGOL \\ Universitat Jaume I, Castellón
}

Aprender a transformar pacíficamente los conflictos es uno de los grandes retos de cualquier sociedad. Hace tiempo ya que sabemos que la conflictividad es una característica inherente al ser humano ${ }^{1}$, sin embargo se encuentra en nuestro ámbito de libertad y responsabilidad cómo abordamos o transformamos los conflictos, con medios violentos o con alternativas pacíficas. Es un gran reto que sólo puede ser abordado desde la interculturalidad y la interdisciplinariedad, ninguna cultura ni disciplina tienen en exclusiva la patente de la solución. Para construir esa cultura de paz que sostenga y promueva la transformación pacífica de conflictos será interesante escuchar todas las aportaciones, todas las voces. Una voz desde la que podemos aprender en ese camino es la de las mujeres, socializadas a lo largo de la historia en el rol de cuidadoras. En este artículo presentaré los indicadores que la ética del cuidado nos aporta para transformar pacíficamente los conflictos entre los seres humanos.

Dividiré mi análisis en tres partes. En la primera haré una introducción a la teoría de los conflictos, disciplina con entidad propia en el marco de la Investigación para la $\operatorname{Paz}^{2}$ y a la dimensión que la categoría género puede jugar en la comprensión de los conflictos. En segundo lugar abordaré la aportación del cuidado a la transformación de conflictos en tres aspectos: la atención a la multiplicidad, la no existencia de ganadores o perdedores y la prioridad en la atención a las necesidades y no en la aplicación de castigos. Finalmente expondré la conexión de la ética del cuidado con la teoría de la noviolencia.

1. Kant ya se refirió a esa inherente conflictividad humana, con el concepto insociable sociabilidad, que además incluía la aceptación de la dimensión creativa-positiva que puede incluir un conflicto. KANT, Inmanuel: Filosofía de la Historia, México, Fondo de Cultura Económica, 1985, pp. 46-50.

2. La Investigación para la Paz incluye estudios tan heterogéneos como la teoría de conflictos, la teoría oficial y teoría crítica del desarrollo, el derecho internacional, estudios sobre la pobreza, la educación para la paz o la interculturalidad, entre otros. Sin embargo la teoría de conflictos juega un papel protagonista en el objetivo común de crear una cultura de paz. Ver FISAS, Vicenç: Cultura de paz y gestión de conflictos, Barcelona, Icaria, 1998. 


\section{LA TEORÍA DE LOS CONFLICTOS Y EL GÉNERO COMO CATEGORÍA DE ANÁLISIS}

La Investigación para la Paz ha estado tradicionalmente unida a los estudios sobre la guerra y los conflictos, sobre todo en sus inicios. Podemos distinguir dentro de la investigación para la paz dos etapas que podríamos calificar como la vieja agenda y la nueva agenda de los estudios para la paz. Uno de los criterios en los que se basa esta clasificación consiste en la mayor o menor relación de los estudios para la paz con los estudios sobre la guerra y los conflictos en general.

La vieja agenda de los estudios para la paz tiene una clara conexión con los estudios sobre la guerra y los conflictos. De hecho el nacimiento de los estudios para la paz está ligado casi de forma indisoluble a los estudios sobre la guerra ${ }^{3}$.

Mientras que en la vieja agenda de la investigación para la paz el tema principal de investigación es la paz negativa (las causas de la guerra, la resolución de conflictos, etc.) en la nueva agenda de la investigación para la paz el tema principal de investigación es la paz positiva (derechos humanos, globalidad y ecología, bienestar económico, noviolencia, etc.). Pasamos de estudiar lo mal que está todo a plantear nuevas opciones de futuro, posibles alternativas, a plantearnos el contenido que una cultura para la paz debería tener.

A pesar de esta diferencia en los temas de estudio, en la actual investigación para la paz todavía siguen en pie estudios sobre la guerra y los conflictos.

Paralelamente a esta evolución con respecto a la vinculación de la investigación para la paz con el estudio de los conflictos, también existe una evolución interna a los estudios sobre conflictos respecto a la forma de entenderlos. Esta evolución puede resumirse básicamente diciendo que se ha pasado de la idea de resolución de conflictos a la de transformación de estos. Como muy bien afirma Vinyamata ${ }^{4}$,

«El conflicto, en sí mismo, no tiene una significación peyorativa; podrá ser nega-

tivo o positivo, dependerá del valor que le asignemos y, sobre todo, del uso que le demos a través de su gestión».

Por eso mismo es más adecuado hablar de transformación que de resolución, ya que los conflictos son inherentes a la actividad humana y social. Nos interesa ante todo aprender a manejarnos en los conflictos, aprender formas pacíficas y justas de transformarlos que nos permitan ver los conflictos más como una oportunidad de crecimiento y mejora que como un mal a extirpar.

3. Los primeros estudios para la paz también son conocidos como estudios de polemología, es decir, estudios sobre la guerra, la actual investigación para la paz, sin embargo, se centra más en la irenología, es decir, en el análisis de los contenidos mismos que garantizan la existencia de una cultura para la paz.

4. UnYAmata, Camp E.: Manual de prevención y resolución de conflictos. Conciliación, mediación, negociación, Barcelona, Ariel, 1999, p. 27. 
Uno de los principales objetivos de los estudios para la paz es reconstruir, reconocer y cultivar las formas pacíficas de transformación de conflictos. Como Margaret Mead o Malinowski afirman, la agresividad es una forma de responder a los conflictos en determinadas sociedades, pero no en todas. Lamentablemente la cultura occidental se caracteriza por la agresividad y la violencia a la hora de resolver los conflictos. Además esta tendencia viene soportada y promovida por los medios de comunicación, especialmente el cine y la televisión.

Desde la investigación para la paz se considera necesario (además de urgente) aprender y promover formas alternativas de transformar conflictos. Con tal fin, una propuesta sería escuchar y aprender de aquellos que tradicionalmente han estado excluidos en la sociedad occidental: mujeres ${ }^{5}$ y culturas no occidentales, por ejemplo. No estoy afirmando con esto que todas las mujeres y todas las culturas no occidentales sean pacíficas por naturaleza, lo cual sería caer en un esencialismo, sino que es moralmente deseable reconocer y valorar todas las experiencias, ya que sólo entre todos podemos aprender formas más creativas y positivas de transformación de conflictos.

Es en este sentido es en el que la ética del cuidado nos puede ayudar a reconstruir habilidades pacíficas para abordar los conflictos.

La ética del cuidado está muy en relación con la importancia de potenciar los vínculos personales, elemento que disminuye la aparición de conflictos y facilita la transformación de los existentes. Carol Gilligan describe esta voz en su famoso libro In a Different Voice cuando tratando de aplicar la escala del desarrollo moral de Kohlberg a la experiencia de las mujeres descubrió unas diferencias básicas producidas por los distintos roles que la sociedad asigna a hombres y mujeres ${ }^{6}$.

En la segunda parte de este artículo voy a señalar algunas de las contribuciones en las que la ética del cuidado nos puede ayudar a gestionar pacíficamente los conflictos:

1. Atención a la multiplicidad, considerar todo lo que se pueda.

2. No existencia de ganadores o perdedores

3. Prioridad en la atención a las necesidades y no en la aplicación de castigos.

Durante siglos estuvimos convencidos de que la tierra era plana, de ser el centro del universo y ahora creemos lo contrario; el gran giro copernicano, sin embargo, todavía está empezando, desechar la guerra y la violencia como forma de resolver los conflictos para redescubrir la grandeza de transformar los conflictos pacíficamente.

Antes de pasar a analizar cada uno de los aspectos en los que la ética del cuidado nos aporta habilidades para transformar pacíficamente los conflictos,

5. El punto j del Programa de Acción de la UNESCO sobre una Cultura de Paz propone, en este sentido, "promover una mayor participación de la mujer en la prevención y la solución de conflictos» (NACIONES UNIDAS: «53/243 Declaración y Programa de Acción sobre una Cultura de Paz", Asamblea General, 6 de octubre de 1999, punto 16, p. 11).

6. Glligan, Carol: La moral y la teoría. Psicología del desarrollo femenino, México, Fondo de Cultura Económica, 1986. 
me gustaría aclarar y explicitar por qué he enmarcado la ética del cuidado como una aportación de las mujeres a la gestión pacífica de conflictos.

Si abordamos el tema de los conflictos tomando en consideración la categoría de género descubriremos algunos datos interesantes. Por ejemplo, que «la mayor parte de los hechos violentos, de violencia directa, son cometidos por varones» " o que "los hombres son también más sensibles que las mujeres a aquellos factores ambientales que ejercen una influencia importante en la conducta antisocial» ${ }^{8}$.

¿Son los hombres intrínsecamente violentos?, ¿son las mujeres más pacíficas por naturaleza? Rotundamente no.

La génesis de la ética del cuidado se encuentra en el análisis de la experiencia moral de mujeres y en el reconocimiento de rasgos diferenciadores con respecto a la experiencia moral de los hombres. Concretamente, las mujeres parecen dar mayor importancia a las relaciones interpersonales y a los individuos concretos, mientras que los hombres dan mayor importancia al cumplimiento de las normas y a ideales de carácter más abstracto. Esta diferencia no se debe a cuestiones biológicas sino a una diferente socialización y construcción de los roles de género. Por su carácter de construcción social no puede generalizarse a todos los individuos, lo cual sería caer en un esencialismo, tanto en el caso de las mujeres como en el de los hombres.

Por este motivo, al hablar de las aportaciones que las mujeres pueden realizar al campo de la transformación de conflictos, debemos tener en claro de qué hablamos. No se trata de que las mujeres en sí mismas, por razones de sexo, sean más pacíficas y por ello podamos aprender de ellas. Realmente, la aportación viene de ciertos rasgos del comportamiento que la sociedad espera en las mujeres.

«Puesto que la moralidad está fuertemente ligada con el problema de la agresión -un área donde las diferencias de sexo son incontestables- puede ser de particular interés para ambos sexos explorar en qué forma las experiencias de las mujeres iluminan la psicología de las estrategias no violentas de resolución de conflictos»?.

Según Sara Ruddick, el conflicto es una parte de la vida maternal diaria. Una madre se encuentra a sí misma enfrentada con sus hijos, con un mundo exterior indiferente con ella y sus intereses, con un hombre u otros adultos en su hogar, con los enemigos de sus hijos. Ella es espectadora y árbitra de las batallas de sus hijos entre sí y sus compañeros. No es sorprendente, entonces, que el pensamiento maternal haya articulado una teoría del conflicto concordante con los

7. MAGALLón, Carmen: «Sostener la vida, producir la muerte: estereotipos de género y violencia», en Vicenç Fisas (ed.): El sexo de la violencia, Barcelona, Icaria, 1998, p. 93.

8. Ibíd., p. 8.

9. Glligan, Carol: «Reply to Critics», en M.J. Larrabee (ed.): An Ethic of Care, London, Routledge, 1993, p. 214. 
objetivos de la práctica maternal. Esta teoría es, en muchas formas, congruente con el pacifismo ${ }^{10}$.

Como todos sabemos, y las investigaciones feministas han analizado y denunciado, el rol de género impuesto en la mujer a través de la socialización, ha servido para anclarla en la esfera privada, en el ámbito familiar, al cuidado de niños, ancianos y enfermos. Un rol de género con el que se ha apartado a las mujeres del ámbito público, de la política, de la cultura e incluso de la historia.

Si bien esta situación es deplorable y debemos hacer todo lo que esté en nuestras manos para modificarla, no debemos rechazar las tareas de cuidado per se y los valores y sentimientos que lo envuelven como malditos, no está ahí la solución al problema. Debemos adoptar este valor, reconstruirlo y educarnos en él tanto hombres como mujeres, debemos des-generizarlo. Conseguir que el cuidado sea un valor y una responsabilidad de todos los seres humanos y no meramente el rol de género atribuido a las mujeres. Teniendo en cuenta que los roles de género son fruto de una construcción social que se ha dado históricamente y mantenido mediante la socialización, se encuentra en nuestras manos transformarlos.

\section{CONTRIBUCIONES DE LA ÉTICA DEL CUIDADO A LA TRANSFORMACIÓN PACÍFICA DE CONFLICTOS}

\subsection{La atención a la multiplicidad, considerar todo lo que se pueda}

Con respecto a la atención a la multiplicidad, la ética del cuidado nos aporta una habilidad muy valiosa en la transformación de conflictos. Ante situaciones conflictivas mostramos a menudo tendencias maniqueístas de considerar las cosas dicotómicamente: el bueno y el malo, la víctima y el verdugo, el bien y el mal. Afortunadamente la realidad no es tan sencilla, sino mucho más compleja.

Tzvetan Todorov en su libro Frente al Limite distingue entre virtudes heroicas y virtudes cotidianas ${ }^{11}$.

Las virtudes heroicas las identificamos con aquellas que, valga la redundancia, posee el héroe: valentía, vida solitaria y sin responsabilidades familiares, ausencia de miedo a la muerte, capacidad de arriesgar la vida por un ideal, por una bandera...

Las virtudes cotidianas por el contrario, aunque no han sido tan veneradas por la literatura, son propias no ya de héroes sino de gente sencilla (ni héroes ni santos). Entrega, cuidado, responsabilidades familiares, preocupación no ya por una idea abstracta sino por otros individuos concretos, ésas son las características de la mayoría de la humanidad.

10. RUDDICK, Sara: «Preservative Love and Military Destruction: Some Reflections on Mothering and Peace», en M. Humm (ed): Feminism, A Reader, New York, Harvester Wheatsheaf, 1992, p. 300.

11. TODOROV, Tzvetan: Frente al Limite, Madrid, Siglo XXI, 1993. 
La historia nos demuestra que las virtudes heroicas nos llevan a guerras, a muerte y desolación. Quizá ha llegado ya la hora de reconocer aquellas virtudes cotidianas que perpetúan y hacen más agradable la vida día a día.

«El mundo de los héroes -y ahí es, tal vez, donde reside su debilidad- es un mundo unidimensional que no comporta más que dos términos opuestos: nosotros y ellos, amigo y enemigo, valor y cobardía, héroe y traidor, negro y blanco» ${ }^{12}$.

La ética del cuidado se opone desde esta perspectiva a la visión heroica. La moral desde Carol Gilligan,

"Consiste en dedicar tiempo y energía para considerarlo todo. Decidir sin cuidado o deprisa sobre la base de uno o dos factores cuando sabemos que hay otras cosas que son importantes y serán afectadas: eso es inmoral. La forma moral de tomar decisiones es considerar todo lo que se pueda, todo lo que se sepa» ${ }^{13}$.

Debemos tener en cuenta también otros puntos de vista y así, a través del diálogo, tomar en consideración otras voces,

"La sensibilidad a las necesidades de los demás y el asumir responsabilidad por cuidar de ellos lleva a las mujeres a escuchar voces distintas de las suyas y a incluir en sus juicios otros puntos de vista. La flaqueza moral de las mujeres se manifiesta en una aparente difusión y confusión de juicio, y resulta así inseparable de la fuerza moral de las mujeres una preocupación predominante por las relaciones y responsabilidades ${ }^{14}$.

Esto muestra lo importante que es para una correcta acción moral el realizar una correcta percepción previa de la situación ${ }^{15}$. Las situaciones son generalmente complejas y multicausales, no tener en consideración todos los aspectos y la mayor parte de voces posibles puede conducir a una penosa acción. Diferentes autores, entre ellos Nussbaum, argumentan que

«Una visión moral adecuada sólo resulta de un esfuerzo mayor, de un escrutinio intenso de la realidad. La atención es ese tipo de escrutinio, y lleva a la observación cuidadosa de las creencias, sentimientos y circunstancias propias y de otros para ver a esta realidad en toda su riqueza y complejidad (...) El lenguaje apropiado para la filosofía moral es precisamente el que se basa en la atención» ${ }^{16}$.

También Todorov critica la visión maniqueísta de la actitud heroica:

"Este sistema de referencias se aviene bien a una situación orientada hacia la muerte pero no hacia la vida. En Varsovia, en 1944, no se enfrentan solamente fuerzas del bien y del mal. Están los rusos y los alemanes, el Ejército del Interior y el Ejército Popular, el gobierno en el exilio y la población civil. En una situación tan compleja, la mejor solución - pero que, en su momento, no es, jay!, más que la menos mala- pasa por la cuidadosa atención a cuanto todos tengan que decir, antes que por

12. Ibíd., p. 20.

13. Glligan, Carol: La moral y la teoria..., op. cit., p. 240.

14. Ibíd., p. 38.

15. SAlles, A. L. F.: «Percepción y emociones en la moralidad», Isegoría, 20 (1999), pp. 217-226.

16. Ibíd., pp. 217-218. 
la fidelidad inquebrantable a su propio ideal. Los valores de la vida, en este sentido, no son absolutos: la vida es diversa, toda situación es heterogénea; las elecciones que uno hace son, así, el resultado no de concesiones o de compromisos cobardes, sino de una toma en consideración de esta multiplicidad ${ }^{17}$.

Atenerse a los mismos principios en diferentes circunstancias puede ser una injusticia. Cuando nos aferramos a los principios, sin atender al contexto, no estamos teniendo en cuenta la complejidad. Ser sensible a un amplio rango de consideraciones morales puede ser a menudo más importante que la adherencia rígida a principios ${ }^{18}$.

Ante situaciones conflictivas puede ser más pacífico establecer un diálogo entre las diferentes partes que ceñirse, sin atender al contexto, a principios universales.

Ser imparcial significa, en teoría, la capacidad de verlo todo, por encima de las perspectivas e intereses particulares. Sin embargo, según la teoría moral feminista la imparcialidad puede ser opresiva ${ }^{19}$.

«Para la filosofía contemporánea poner en cuestión el ideal de imparcialidad lleva a cuestionar la posibilidad misma de la teoría moral $»^{20}$.

El punto de vista imparcial está relacionado con el interés de adoptar principios universales.

«Este deseo es al menos tan viejo como Parménides, y la lógica de la identidad comienza con la antigua noción filosófica de los universales $\aleph^{21}$.

Pero este tipo de razonamiento elimina la otredad de dos formas, la irreductible especificidad de las situaciones y la diferencia entre sujetos morales ${ }^{22}$.

«El sujeto imparcial no necesita reconocer a otros sujetos cuya perspectiva debiera ser tomada en cuenta y con quien pudiera producirse alguna discusión. Por lo que la pretensión de imparcialidad suele dar como resultado el autoritarismo. Al decir de uno mismo que se es imparcial, se recurre a la autoridad para decidir un tema, en lugar de aquellos cuyos intereses y deseos son manifiestos. Desde este punto de vista imparcial, no se necesita consultar con nadie más, debido a que el punto de vista imparcial ya toma en cuenta cualquier perspectiva posible $»^{23}$.

El compromiso con la imparcialidad da como resultado una oposición entre razón y deseo. La imparcialidad requiere que la razón se despoje de todo sentimiento, de toda afectividad. La imparcialidad exige hacer abstracción de

17. Todorov, Tzvetan: Op. cit., p. 20.

18. HLD, V.: Justice and Care: Essential Readings in Feminist Ethics, Colorado, Westview Press, 1995, p. 162.

19. YounG, I. M.: «Imparcialidad y lo cívico público. Algunas implicaciones de las críticas feministas a la teoría moral y política», en S. Benhabib y D. Cornell (eds.): Teoría Feminista y Teoría Crítica, Valencia, Edicions Alfons el Magnànim, 1990, pp. 92-94.

20. Ibíd., p. 93.

21. Ibíd., p. 95

22. Ibíd., p. 95

23. Ibíd., p. 96 
la particularidad del ser corpóreo, de sus necesidades e inclinaciones, y de los sentimientos que se vinculan a la particularidad experimentada ${ }^{24}$. Como los sentimientos y la afectividad parecen ser el elemento diferenciador entre las personas es el elemento que debe obviarse al tomar el punto de vista imparcial.

\subsection{No existencia de ganadores o perdedores}

«Estamos acostumbrados a que en todo conflicto, en toda negociación, una de las partes ha de perder o, en el mejor de los casos, de que uno de los contrincantes ha de ganar más que el otro. Sin embargo, no existe ninguna razón sólida para oponerse a la hipótesis de que todas las partes puedan salir igualmente satisfechas ${ }^{25}$.

Esta afirmación de Vinyamata queda respaldada por Carol Gilligan y la teoría de la ética del cuidado.

Carol Gilligan descubrió una diferencia a la hora de entender los problemas morales entre los hombres y las mujeres (que como ya hemos señalado es de carácter social), distintos modos de entendimiento moral, distintos modos de pensar acerca del conflicto y la elección ${ }^{26}$. Esto queda claramente expresado en la diferente resolución que se da al Dilema de Heinz por un niño y por una niña.

En este dilema un hombre llamado Heinz reflexiona sobre si debe robar o no un medicamento que no puede comprar para salvar la vida de su esposa, la pregunta es ¿debe Heinz robar el medicamento? La respuesta (en esta entrevista de Gilligan) que dio el niño fue clara: «Heinz debe robar el medicamento», el niño ha interpretado el dilema como un conflicto entre los valores de la propiedad y de la vida, claramente deduce que la vida tiene prioridad sobre la propiedad. Sin embargo la respuesta de la niña parecía indecisa, no veía el dilema como un conflicto de valores al que se le puede aplicar una escala ya predeterminada sino como "un relato de relaciones que se extienden en el tiempo» ${ }^{27}$. Trata de llegar a una solución en la que todos queden de algún grado "satisfechos» y en la que no se rompa la conexión entre los tres individuos (la esposa, el marido y el farmacéutico). Desde la perspectiva de la ética del cuidado no tiene porqué existir siempre algún perdedor. El deseo de no dañar a nadie y la esperanza de que en la moral se encuentre una manera de resolver conflictos, en que nadie salga dañado caracteriza el razonamiento moral desde el cuidado ${ }^{28}$.

Además la respuesta del niño surge a partir de una imagen violentada, que ve un mundo de peligrosa confrontación y competitiva explosión, mientras que la niña ve un mundo de atención y protección, una vida vivida con otros.

24. Ibíd., pp. 94-97.

25. UNYAMATA, Camp E.: Op. cit., p. 28.

26. Glligan, Carol: La moral y la teoría..., op.cit., p. 62.

27. Ibíd., p. 55.

28. Ibíd., p. 114. 
"Las frecuentes soluciones, a las que se llega con un ganador y un perdedor (con todos los daños psicológicos y políticos que ello comporta), podrían reemplazarse por aquellas en las que ambas partes son ganadoras» ${ }^{29}$.

La historia nos ha demostrado y sigue demostrando que la aparición de perdedores o culpables en la regulación de conflictos es una fuente de nuevos conflictos. Es, entre otras cosas, una semilla para la venganza, el resentimiento y el odio.

«La cultura de gestión de conflictos que defendemos parte de la base de que los dualismos (ganador-perdedor [...]) comportan nuevos conflictos futuros, y que una buena prevención se inscribe en una buena gestión de los conflictos presentes, abandonando los dualismos y buscando posiciones intermedias» ${ }^{30}$.

\subsection{Prioridad en la atención a las necesidades y no en la aplicación de castigos}

Otra aportación de la ética del cuidado a la transformación pacífica de conflictos es el hecho de que en la reflexión desde el cuidado, ante un conflicto el principal interés o foco de atención es satisfacer la necesidad (de ahí el nombre de ética del cuidado). En cambio, desde la ética de la justicia, aunque los teóricos tienen en cuenta la satisfacción de las necesidades y su importante papel, su foco de atención se dirige a la penalización y regularización de la agresividad. En la ética del cuidado hay una primacía de satisfacer la necesidad frente a castigar la agresividad.

Es un error limitar la moralidad a la resolución de conflictos, o en otras palabras, a la construcción de principios abstractos del bien, lo importante de una ética del cuidado es su orientación hacia la prevención del conflicto. Para prevenir el conflicto, el crimen y la injusticia necesitamos de la ética del cuidado y de su reconocimiento de la importancia de la conexión humana para evitar la injusticia.

Diferentes teóricos de la ética del cuidado como Fiona Robinson, Clement o Tronto, han argumentado la importancia de extender el cuidado más allá de la esfera privada a la esfera pública y política ${ }^{31}$.

El castigo en la ética de la justicia puede llevarnos a crear más violencia, la ética del cuidado trata de suprimir la violencia y cree más importante satisfacer las necesidades que castigar las culpas o sacrificar la vida humana por algún precepto. Summum jus, summa injuria reza la sentencia latina atribuida a Cicerón que advierte de las inequidades que se pueden cometer aplicando la ley de modo demasiado riguroso.

29. CRUZ ROJA ESPAÑOLA: 14 informes técnicos. Seminario sobre formación de monitores de educación para la paz, Madrid, Cruz Roja Española, 1989, p. 130.

30. BuRgueT, M.: El educador como gestor de conflictos, Bilbao, Desclée de Brouwer, 1999, p. 23.

31. RoBInson, F.: Globalizing Care. Ethics, Feminist Theory and International Relations, Oxford, Westview Press, 1999. 
Así resulta muy útil el símil que utiliza Gilligan comparando la actitud de Abraham en contraste con la mujer que se presenta ante Salomón. Abraham no se negó a sacrificar la vida de su hijo para demostrar la integridad y supremacía de su fe. En cambio la mujer que se presenta ante Salomón comprueba su maternidad abandonando la verdad para salvar la vida de su hijo. La ciega disposición a sacrificar personas en aras de la verdad siempre ha sido el peligro de la ética apartada de la vida ${ }^{32}$.

\section{LA ÉTICA DEL CUIDADO Y LA TEORÍA DE LA NOVIOLENCIA ${ }^{33}$}

En este apartado demostraré cómo la ética del cuidado tiene muchos puntos en común con la teoría de la noviolencia y que ambas comparten la misma premisa de partida: la transformación pacífica de los conflictos.

"Mientras que una ética de la justicia procede de la premisa de igualdad-que todos deben ser tratados igualmente-, una ética del cuidado se apoya en la premisa de la no violencia: que no se debe dañar a nadie» ${ }^{34}$.

La teoría de la noviolencia tiene dos características fundamentales. Aunque para muchos ha tenido un claro fruto social a gran escala (Gandhi, Martín Luther King, etc.) para la mayoría de los mortales la noviolencia tiene la capacidad de transformación en lo cercano. Esto no le quita importancia, ni tampoco influencia social y debemos ser conscientes de ello para no sentirnos frustrados por no llegar lejos. Esto sólo nos ayudaría a abandonar antes de hora. La noviolencia parte de un interés por lo más cercano, por las acciones cotidianas, por nuestra actitud en el trabajo, en la familia, con los amigos, con la pareja, con el vecino... Aquí nos sirve el lema de la ecología: «pensar global, actuar local». Otro punto clave de la noviolencia es la perseverancia, pues debe ser una forma de vida y no una serie de actos esporádicos.

Partiendo de estas dos características de toda acción noviolenta, el eje principal que define la teoría de la noviolencia y también la ética del cuidado es la fuerza del amor. El amor ha sido condenado al ostracismo por casi todas las disciplinas científicas, incluso por aquellas que se hacen llamar Ciencias Humanas y Sociales. Sólo desde determinados ámbitos de la psicología, la ética y poco más existe una somera aproximación. Es un reto de los seres humanos reconstruir este valor, aprenderlo y cultivarlo.

«La noviolencia no sólo ha sido un método (...) de lucha para transformar los conflictos, denunciar los niveles existentes de violencia o abordar cambios representativos en las sociedades. Sino que, también, está intentando renovar (...) a otras disciplinas de conocimientos, tales como: la historia (...), la teoría política (...), la sociología (...), la antropología (...), la religión (...), la filosofía ética (con la denominada

32. Glligan, C.: Op. cit., p. 173.

33. Respecto a la relación entre noviolencia y práctica maternal es interesante el libro de RUDDICK, Sara: Maternal Thinking: Toward a Politics of Peace, New York, Women's Press, 1989.

34. Glligan, C.: Op. cit., p. 281. 
ética del cuidado), la economía (...), el feminismo (reforzando especialmente el de la diferencia) y también las llamadas ciencias experimentales $»^{35}$.

Como muy bien apunta Mayor Zaragoza ¿por qué no nos atrevemos a correr el riesgo que toda religión y filosofía importante nos asegura que debemos correr, el riesgo de amary ser amados? ${ }^{36}$ Existen diferentes fuentes desde las que podríamos reconstruir el amor como valor, aunque cabría destacar dos sedes fundamentales.

El primer lugar al que tradicionalmente se ha relegado tanto el amor como otros sentimientos morales a sido el ámbito de lo privado, lo doméstico, lo femenino... Las mujeres tienen mucho que enseñar en este valor, en el valor del cuidado y la ternura. Gandhi mantuvo que había aprendido las técnicas de la noviolencia y de la desobediencia civil de las mujeres, principalmente de las sufragistas británicas. ${ }^{37}$ Valores que tradicionalmente han estado relegados al ámbito doméstico y que han servido como elemento subordinador de la mujer, son reformulados y reconstruidos ahora como prácticas sociales de transformación pacífica de conflictos tanto para el hombre como para la mujer. Estos valores formaban parte de las redes opresivas del sistema género-sexo, ahora se trata, no de rechazarlos sino de recuperarlos para todos los seres humanos.

Históricamente una de las fuentes que más valor e importancia ha dado al amor ha sido la religión. Casi todas las religiones ven en el amor al prójimo el mandamiento principal. La noviolencia se ha nutrido de las grandes religiones como experiencias de paz, de reconciliación y perdón. Aunque existen también experiencias de violencia y de guerra relacionadas con temas religiosos cabe recordar que la paz es un elemento central en los fundamentos y bases teóricas de la religión ${ }^{38}$.

Así, deberíamos hurgar en todas aquellas tradiciones y aportaciones que nos ayuden a reconstruir el valor del amor.

Según Sara Ruddick, la experiencia maternal es una fuente de aprendizaje de habilidades para resolver conflictos de forma no violenta. Más allá de la práctica maternal aparece un pensamiento distintivo que es incompatible con la estrategia militar pero que concuerda con el compromiso pacífico de la noviolencia ${ }^{39}$.

"Tanto en sus casas como fuera de ellas, las mujeres normalmente se experimentan a sí mismas como débiles, sin poder. Ellas son normalmente pobres socialmente, objetos más que agentes de guerras, planes económicos y regímenes políticos. Como otros combatientes sin poder, las madres a menudo recurren a estrategias de noviolencia porque ellas no tienen armas con que dañar: ni rifles, ni efectividad legal ni

35. Iópez MARTíneZ, Mario: «La noviolencia como alternativa política» en F.A. Muñoz, F.A. (ed.): La paz imperfecta, Granada, Ediciones Universidad de Granada, 2001, p. 232.

36. MAYOR ZARAGOZA, Federico: La nueva página, Barcelona, Galaxia Gutenberg, 1994.

37. MAgALLÓN PORTOLÉS, Carmen: «Hombres y mujeres: el sistema sexo-género y sus implicaciones para la paz», Mientras Tanto, 54 (1993), p. 70.

38. CABEDO MANUEL, Salvador: «Religión y paz en Kant», en V. Martínez Guzmán (ed.) Kant: La paz perpetua, doscientos años después, Castellón, Nau Llibres, 1997, pp. 35-51.

39. RUDDICK, Sara: «Preservative Love and Military Destruction..., op. cit., p. 299. 
poder económico. Así es como las madres se enrolan en técnicas no violentas como el ruego, la persuasión, el apaciguamiento, el auto-sacrificio, la negociación y toda una variedad de habilidades emocionales. Cada una de esas técnicas tiene, según Ruddick, un lugar en la coerción pública no violenta» ${ }^{40}$.

Cuando hablamos de la fuerza del amor, nos referimos al amor que siento en el otro en el que veo la esencia del ser humano, la humanidad. Amo en él la vida, el valor de ser humano, de ser mi igual y formar parte de una unidad. Este tipo de amor también debe darse con la naturaleza, con el sol, las estrellas. Es el amor por lo que existe, por aquello que nos ha dado la vida y por aquello por lo que vale la pena morir ${ }^{41}$.

Este concepto amplio de amor incluye el amor por la naturaleza, por otros y por nosotros mismos. Este amor debe empezar por uno mismo. Uno tiene que quererse a si mismo para así poder amar mejor a los demás.

Al igual que la violencia crea un círculo vicioso, el amor crea un círculo virtuoso que revierte en más amor. Por eso podemos considerar el amor como un método de construir una Cultura para la Paz. La noviolencia dice que podemos romper con la espiral de la violencia apostando por la fuerza del amor.

Este amor también está muy conectado con la necesidad de reconocimiento que todos los seres humanos necesitamos. Si a un niño pequeño le mostramos amor y reconocimiento (le cuidamos) crece con un autoconcepto sano y se siente seguro de sí mismo, capaz de plantear alternativas y soluciones a la vida. Es muy importante la autoestima y tener un buen autoconcepto para poder plantear alternativas a los conflictos. Esta autoestima sólo puede desarrollarse a través del amor. Cuando amamos a alguien, con ese amor que recibe aprende a amarse también a sí mismo y eso facilitará que ame mejor a los demás, por eso hablamos del círculo virtuoso del amor.

Esta idea del amor como reconocimiento también nos lleva a la idea de que la capacidad del noviolento está en encontrar la parte buena del otro y tratar de potenciarla. Está claro que no somos unos ángeles ni tampoco unos demonios, hasta la persona más brusca y cruel tiene aspectos positivos. La tarea más importante del noviolento es encontrar esos aspectos y saber potenciarlos, valorarlos. Se avanza más potenciando los valores buenos que criticando los aspectos negativos.

Por todo ello abogamos por el concepto de ahimsa para definir la noviolencia. En Occidente hablamos de noviolencia como negación de la violencia, sin embargo ahimsa, no es solamente noviolencia sino que sería además la fuerza del amor, del cuidado y la ternura ${ }^{42}$.

40. Ibíd.., p. 301.

41. "Conozco mil razones por las que vale la pena morir pero no conozco ninguna por la que valga la pena matar» famosa frase atribuida a Mahatma Gandhi.

42. MARTínez GuZMÁN, Vicent: Filosofía para hacer las paces, Barcelona, Icaria, 2001, p. 121. 
La ética del cuidado: contribuciones a una transformación pacífica de los conflictos

\section{CONCLUSIÓN}

El cuidado de los demás ha sido un rol atribuido históricamente a las mujeres, un rol que muchas veces ha servido para tenerla sometida al ámbito privado de la crianza de los hijos, del cuidado de enfermos y ancianos.

Diferentes investigaciones, entre las que destacan las realizadas por Carol Gilligan y Sara Ruddick, han demostrado que el rol del cuidar implica el desarrollo de determinadas aptitudes muy afines a la paz: la paciencia, la ternura, la responsabilidad, la empatía, la perseverancia, la prudencia y la tolerancia, entre otras son componentes del cuidar. Aún más, desde el cuidar aprendemos también indicadores de transformación pacífica de conflictos: tener en cuenta todos los puntos de vista, todas las voces y dedicar el tiempo necesario en nuestras decisiones morales, buscando formas de que nadie salga dañado y en las que lo prioritario sea la satisfacción de necesidades.

La propuesta será pues desgenerizar el cuidar como rol exclusivo de las mujeres para transformarlo en un valor humano. Compartir la crianza de los hijos con los hombres no es sólo necesario para conseguir la igualdad sino también es un componente de nuestro objetivo por construir una cultura de paz. Es necesario romper con la responsabilidad tradicionalmente acuñada a los hombres como destructores de las vidas humanas oficialmente etiquetadas como enemigos (el estereotipo del buen soldado), un rol obsoleto y que sólo ha contribuido al desarrollo de una cultura de la violencia ${ }^{43}$. La mujer no es más pacífica por naturaleza, hombres y mujeres nacemos con las mismas capacidades, ahora bien el rol que la sociedad nos atribuye y en el que nos socializamos hace desarrollar en nosotros distintas habilidades. Propuestas actuales como la escuela coeducativa o compartir la crianza de los hijos son algunos de los caminos que podemos emprender en este objetivo de conseguir unos roles de género más justos y pacíficos. El cuidado de los demás es un excelente aprendizaje de transformación pacífica de conflictos, de enriquecimiento moral y afectivo. Vale la pena compartirlo.

43. Baier analiza los dos estereotipos sobre los que se construyen los roles de género: el estereotipo de mujer es la buena madre, mientras que el estereotipo de hombre es el buen soldado. LARRABEe, M.J.: An Ethic of Care, London, Routledge, 1993, p. 26. 